\title{
Idiopathic Pruritus Ani and Acute Perianal Dermatitis
}

\author{
Adrián E. Ortega, MD, FASCRS ${ }^{1}$ Xavier Delgadillo, MD, EBSQ-C² \\ ${ }^{1}$ Keck School of Medicine at the University of Southern California, Los \\ Angeles, California \\ ${ }^{2}$ Centre Médico Chirurgical Volta, La Chaux-de-Fonds, Switzerland \\ Address for correspondence Adrián E. Ortega, MD, FASCRS, \\ LAC+USC Medical Center, Clinic Tower, Room 6A231-A, 1200 N. State \\ Street, Los Angeles, CA 90033 (e-mail: sccowboy78@gmail.com).
}

Clin Colon Rectal Surg 2019;32:327-332.
Abstract
Keywords
- idiopathic
- pruritus ani
- contact
- irritant
- dermatitis
- diagnosis
- treatment

This article reviews the etiologies, pathophysiology, clinical evaluation, and treatment of idiopathic pruritus ani and perianal dermatitis. It underscores a practical approach to each of these common proctologic conditions.

\section{Idiopathic Pruritus Ani}

Pruritus ani can be an important symptom secondary to specific disease processes. Inflammatory diseases of the skin, infections, infestations, premalignant and malignant neoplasms, and anorectal and systemic diseases may manifest with perianal itching. More commonly, there is no diseasespecific etiology. This clinical scenario is termed idiopathic pruritus ani. This nonspecific type is responsible for 50 to $90 \%$ of cases. It affects 1 to $5 \%$ of the population. ${ }^{1}$

The precise inciting event underlying idiopathic pruritus ani is difficult to identify. There are two noteworthy exceptions. First, a careful history of an individual's postevacuation hygiene may identify patterns of inadequate, excessive, and/ or aggressive hygiene. Physical examination readily confirms patients with poor hygiene ( $\mathbf{- F i g .} \mathbf{1}$ ).

Irrespective of the inciting events, a progressive cycle of itching and scratching ensues. Several mitigating factors have been identified and may be operant before or during the itch-scratch cycle. These are listed in the table within - Fig. 2.

The most common denominator linking most cases of idiopathic pruritus ani is fecal contamination. Bowel movements present both irritants and allergens to the perianal tissues. Feces and bacterial enzymes potentiate irritation, itching, and inflammation. This effect has been substantiated by positive skin patch tests using autologous feces. ${ }^{1}$

Issue Theme Perianal Dermatology; Guest Editors: Adrián E. Ortega, MD, FASCRS, and Xavier Delgadillo, MD, EBSQ-C
Diverse consumptive and dietary factors operant in pruritus ani may have a common denominator $-\mathrm{pH}$ alteration of mucous within the hindgut. Seepage has an irritant effect well known in patients with impaired fecal continence as well as in diarrheal states. Flatulence represents the microaerosolized delivery of mucofeculent material to the perianal tissues. Therefore, this normal activity may exacerbate the itch-scratch cycle. Occult mucofeculent leakage has also been implicated as an operant factor. Patients with idiopathic pruritus ani may demonstrate lower thresholds for intersphincteric relaxation as part of the rectoanal inhibitory reflex. ${ }^{2,3}$ An individual's efforts to improve their hygiene with soaps and other remedies may escalate the symptoms. Shaving the anal margin can incite intense pruritus ani. Topical lidocaine can sensitize the tissues or produce a chemical dermatitis when used chronically.

The symptoms and signs associated with idiopathic pruritus ani are varied. Itching is the hallmark presentation. It may vary from mild to severe. Scratching can be remarkable satisfying for some patients. Asleep patients are unable to resists the urge to scratch. When injury to the soft tissues occurs, scratching becomes painful. Severe burning, pain, and bleeding may ensue. Some patients report stress and anxiety as associated symptoms accentuating pruritus ani.

Many individuals have no overt signs of injury. More extensive injury results in redness, abrasions, and excoriations ( - Fig. 3). Long-standing pruritus ani produces lichen
Copyright (c 2019 by Thieme Medical Publishers, Inc., 333 Seventh Avenue, New York, NY 10001, USA. Tel: +1(212) 584-4662.
DOI https://doi.org/ 10.1055/s-0039-1687827. ISSN 1531-0043. 


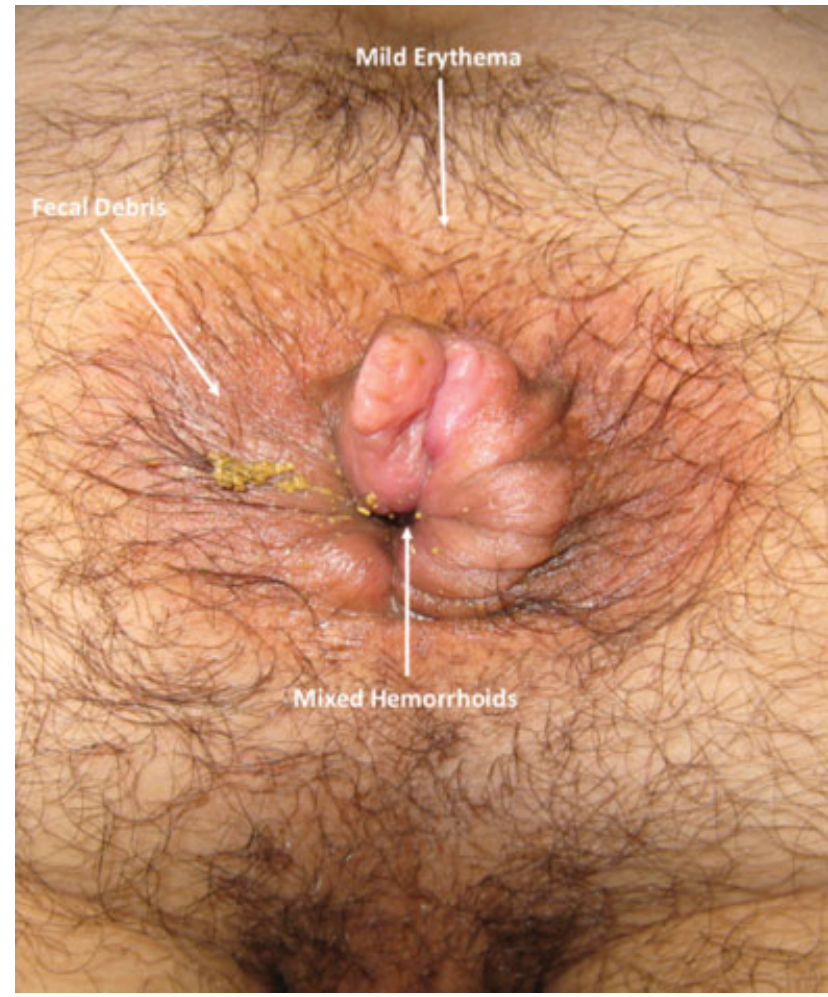

Fig. 1 Illustrates fecal debris, mild erythema, and mixed hemorrhoids at the anal verge of a male with pruritus ani. (This image is provided courtesy of A. Ortega.)

simplex. The tissues within the anal margin are thickened and the rugal folds are deeper and/or superficial fissuring (radial cracks) of the skin may be apparent in this setting (-Fig.4). Frank ulceration of the skin is more concerning and requires definitive diagnosis with biopsy and pathologic confirmation.

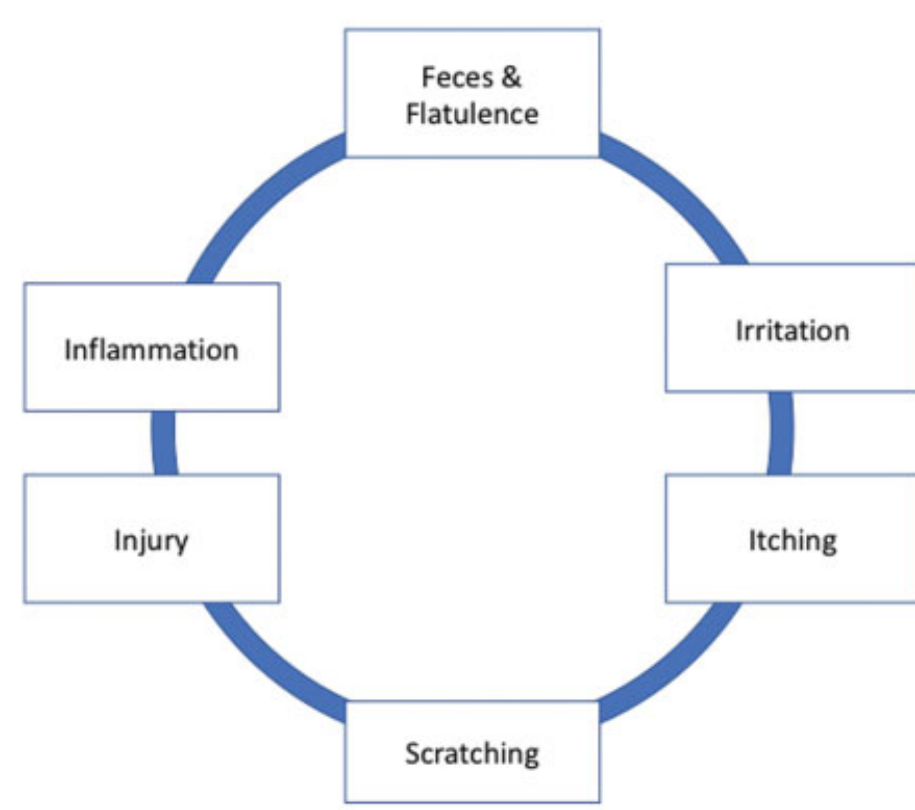

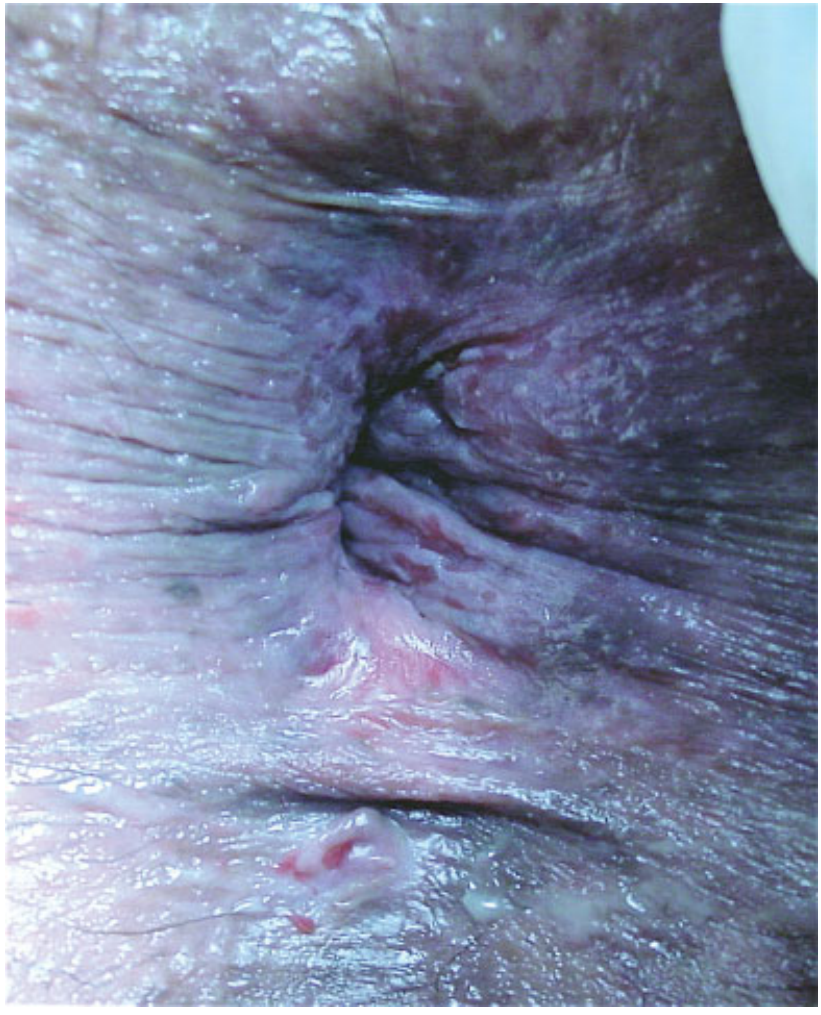

Fig. 3 Severely excoriated and macerated tissues are evident within the anal margin representative of one extreme of the itch-scratch cycle. (This image is provided courtesy of A. Ortega.)

The traditional principles of medical history acquisition are operant-onset, duration, and associated symptoms. Concurrent medical conditions, past surgical procedures, and medication history are important. The affliction of other family members with the same symptoms is an important exclusion. The specific hygiene practices can help distinguish
Mitigating factors:

- Behaviors

- Consumptions

- Ingestions

- Other

Fig. 2 The itch-scratch cycle of idiopathic pruritus ani and potentially inciting and mitigating factors is illustrated. These events are progressive clinically constituting a vicious cycle. 


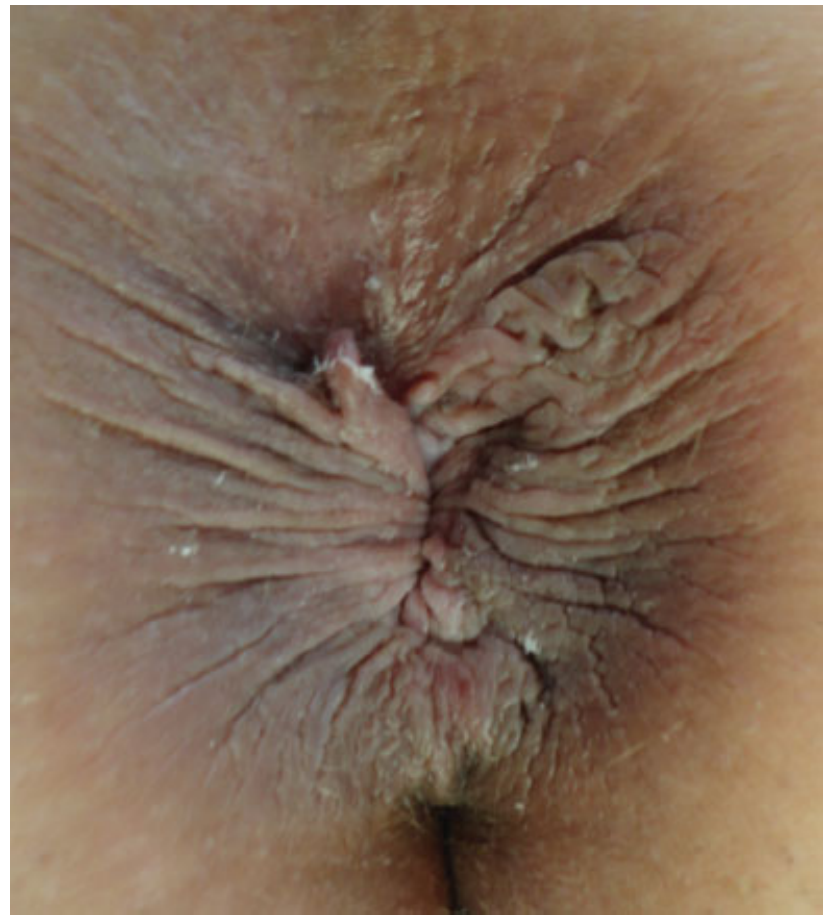

Fig. 4 Lichen simplex is the chronic presentation of pruritus ani. Thickening of the anal verge tissues and deepening of the rugal folds may be apparent as well as concentric superficial radial fissuring. (This image is provided courtesy of I. Khubchandani.)

between insufficient versus aggressive or excessive practices. Documentation of alcohol, tobacco, and caffeine, and similar compounds is important. Other dietary factors should be considered including those that alter the $\mathrm{pH}$ of colorectal mucous or increase its delivery by flatulence.

Most patients with pruritus ani have a nonspecific etiology. One of the principle goals of physical examination is the differentiation between disease-specific and idiopathic causes. The latter is by exclusion of the former which requires a high degree of acuity in physical diagnosis. Most patients with idiopathic pruritus ani will have a normal or relatively normal topographic physical examination. The presence of excoriations or lichen simplex represents more severe or more chronic presentations. The presence of discoloration, erythema, ulcers, or masses should shift away the clinicians' focus to disease-based etiologies versus the more common idiopathic, nonspecific causes. Digital rectal and ano-proctoscopic examinations are important adjuncts to the topographic examination of the perianal tissues. All positions are used in physical examination including prone jackknife, lateral decubitus, and lithotomy. The most important element is that both the patient and the examining position should be comfortable in conducting a gentle, albeit rigorous examination. The identification of common associated findings including anal fistula, hemorrhoids, and skin tags may factor in the therapeutic decision making significantly. - Fig. 5 illustrates a Whitehead deformity following hemorrhoidal surgery resulting in a chronically wet anus and pruritus.

Target lock on the perianal region should be avoided. Disease-specific conditions often affect the genital regions as

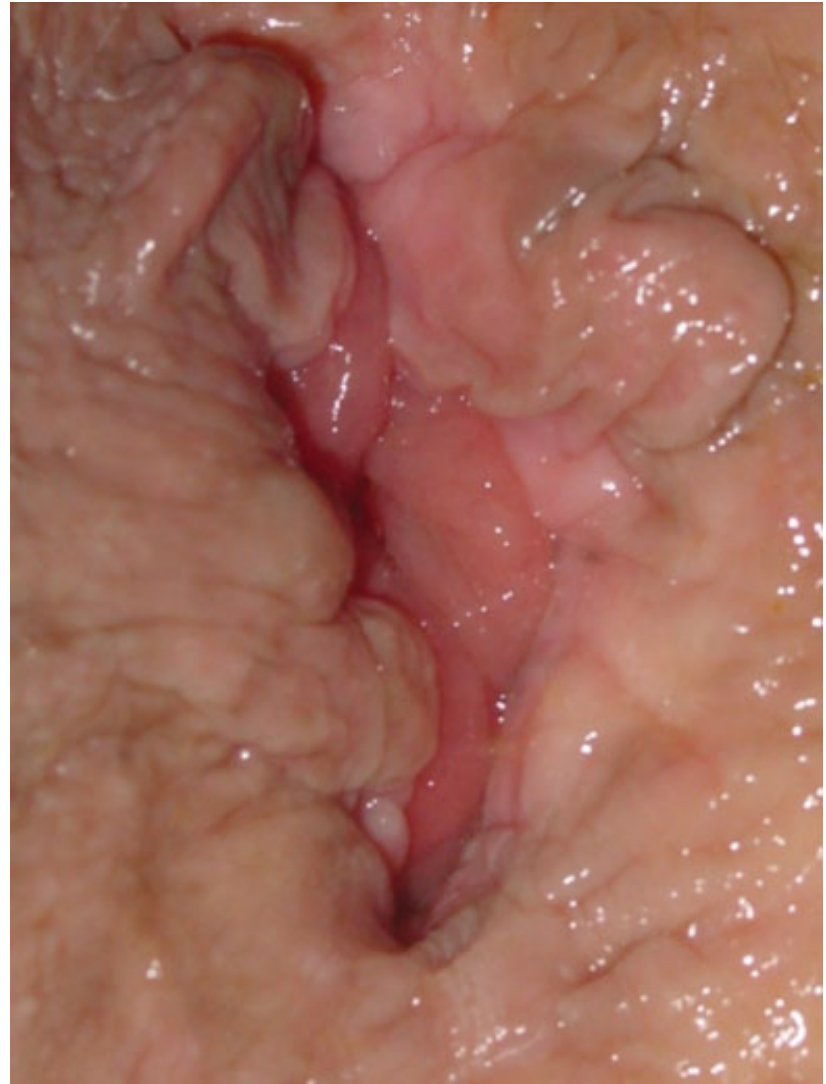

Fig. 5 The Whitehead deformity following hemorrhoid surgery exemplifies a surgical cause of pruritus ani diagnosed on physical examination. (This image is provided courtesy of A. Ortega.)

well as the perineal and perianal areas. Occasionally, similar lesions are seen in other distant locations heightening the suspicion of a systemic disease process.

Most idiopathic processes in medicine are diagnosis of exclusion. However, idiopathic pruritus ani is so overwhelmingly more common than disease-specific causes of anal itching that it is not practical to systematically exclude primary etiologies. Adjunctive tests should be used selectively based on comorbidities, family, and social history, as well as physical findings. Multiple family members with pruritus ani are uncommon in adult patients. However, the exclusion of pinworm infection is reasonable in this context. Diabetic patients with violaceous pinkish discoloration of the anal verge and surrounding tissues merit exclusion of fungal infections. Discreet unilateral or persistent lesions require biopsy. Skin patch testing to autologous feces has been described. Stool pH can be tested. However, positive skin or stool tests do not materially change management.

\section{A Practical Clinical Approach to Idiopathic Pruritus Ani}

The primary goal in management of idiopathic pruritus ani is breaking the itch-scratch cycle. This process begins with gentle but thorough hygiene. Wiping with sanitary tissue can be minimized by encouraging hydrotherapy following bowel movements. The use of sitz baths and bidets are well described but perhaps unpractical for many patients. Cleansing the area with a portable shower head is a practical 
alternative. Additional measures include the avoidance of soaps and other topical agents that can further dry or injure an already sensitized area. Patients should be counseled regarding the behaviors and consumptions known to aggravate pruritus ani. Avoidance or abstinence from agents including alcohol, tobacco, and caffeine should be advised. Dietary exclusion of tomatoes, citrus, and spicy ingestions may help some patients. Dietary manipulation with probiotics is reasonable to reduce flatulence and the delivery of aerosolized mucofeculent matter to the affected region. These types of behavior modification should be sufficient in most patients with idiopathic pruritus ani.

Second-line treatments include short course topical steroids. Long-term use is ill-advised secondary to atrophy of the skin and its secretory glands thereby resulting in intractable pruritus ani. Oral diphenhydramine may be prescribed at bedtime-particularly in those individuals most affected during sleep. Combination of creams with steroid, antifungal, and antibacterial components may produce dramatic improvement in some individuals. When all measures fail, punch biopsy is warranted to exclude adenomatous and squamous premalignant neoplasia.

Patients with intractable diarrhea or altered anal continence may require barrier creams to protect the perianal integumentary tissues. Zinc oxide ointments are useful for this purpose. Third-line treatments particularly for medically refractory pruritus ani include topical capsaicin ointment, ${ }^{4}$ tacrolimus ointment, ${ }^{5}$ and intradermal injection of $1 \%$ methylene blue alone or in combination with $0.5 \%$ lidocaine has been reported. ${ }^{6}$ The results of these approaches are generally favorable in the treatment of intractable pruritus ani.
Pruritus ani may be a prominent feature in patients with symptomatic hemorrhoids. Hemorrhoidectomy may not necessarily relieve symptoms. Therefore, pruritus ani is not an indication for hemorrhoidectomy. However, prominent skin tags can be associated with difficult hygiene, irritation, and intermittent swelling, as well as discomfort. Excision of skin tags may be considered if conservative hygienic measures fail to provide relief ( - Fig. $\mathbf{6}$ ).

\section{Acute Perianal Dermatitis}

The causes of acute perianal dermatitis are divided into two groups: (1) infectious (transmitted) and (2) contact (irritant) dermatitides. Infectious dermatitis may result from close contact transmission between individuals or environmental exposures. Viral, bacterial, mycotic, and protozoal microbes may be implicated. The clinical approach for each infectious etiology is addressed subsequently in this issue.

\section{Irritant-Contact Dermatitis}

Irritant and contact dermatitides on the anal region are a localized acute inflammatory reaction of the perianal skin caused by contact with a foreign substance. As Morris-Jones et al proposed, ${ }^{7}$ this inflammatory condition of skin can be divided into three forms caused by chemical irritants, physical irritants, and a rare form caused by special products. Common chemical irritants implicated include: alcohol, xylene, turpentine, esters, acetone, and ketones. Metalworking fluids such as neat oils and water-based metalworking fluids with surfactants have been implicated. Latex including nonoxinol-9 used in spermicidal jellies and some condom lubricants, kerosene;

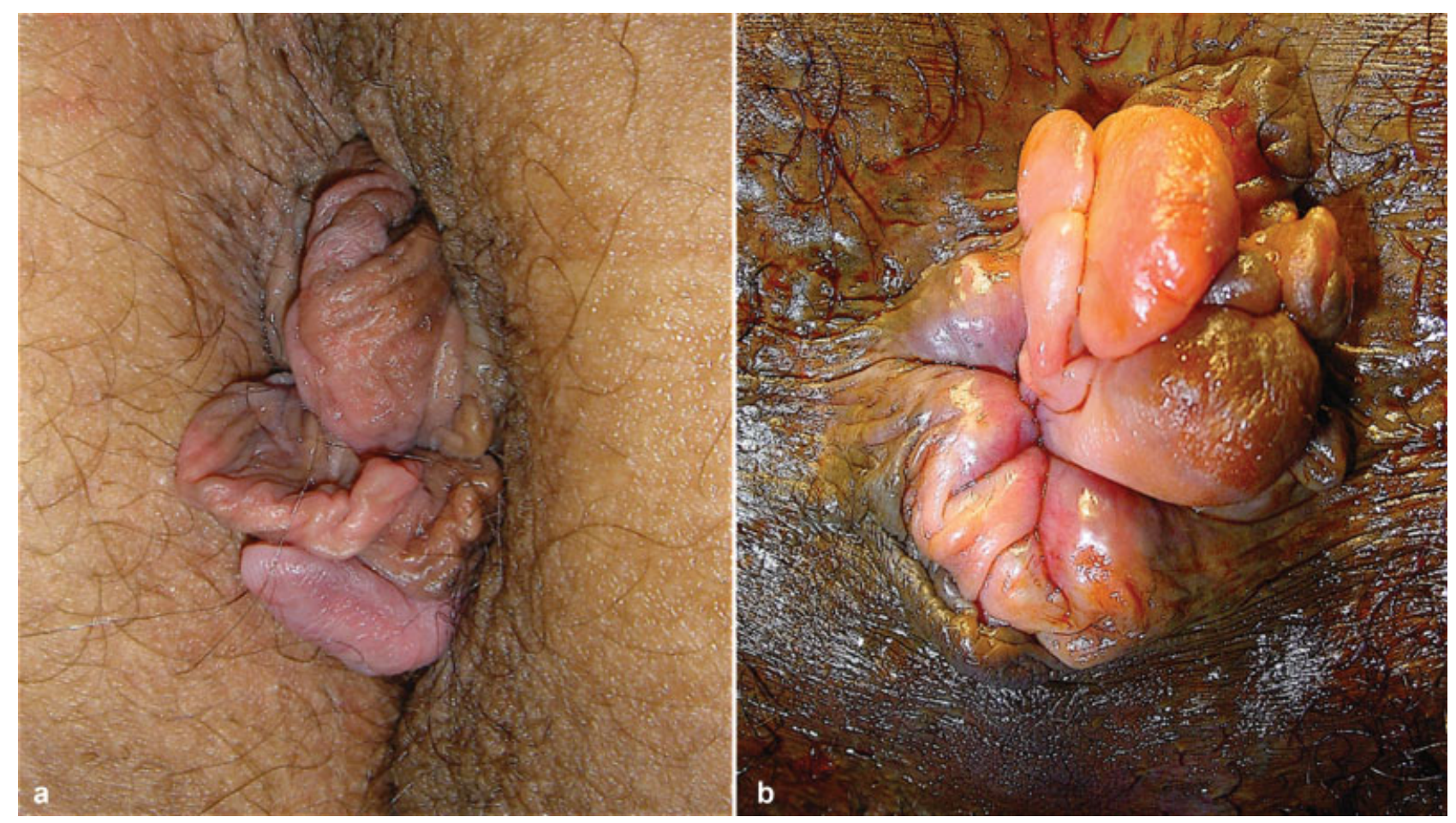

Fig. 6 (a) Extensive appendages (skin tags) within the anal verge. (b) A conglomerate of hypertrophic papillae, papillomas, and skin tags is in evidence. Both examples when associated with pruritus ani may benefit from surgical resection to facilitate local hygiene. (These images are provided courtesy of A. Ortega.) 
ethylene oxide; surfactants in topical medications and cosmetics (sodium lauryl sulfate); and many of drain cleaners, strong soaps with lye residues. Physical irritant contact dermatitis may be caused allegedly by low humidity from air conditioning. Contact with poorly breathing fabrics is anecdotally implicated. Special irritant substances must be defined by a precise history of contact with a possible allergen including benzocaine, lanolin, Peru balsam, cocoa butter, hamamelis, iodine solutions, alcamphor, resorcinol, chamomile, antihistamines, neomycin, and phenol.

Eczema is a general term describing an allergen-induced skin rash. It affects the superficial layers of the skin. Like other forms of contact dermatitis, it persists as long as an individual is exposed to the offending agent. It differs from urticaria (hives)a rash that appears within minutes of exposure and then fades away within minutes to hours. The manifestations of eczema may be self-limited, recurrent, and commonly lifelong (atopic dermatitis). Contact dermatitis may result in large, burning, and itchy rashes. Dermatological changes include erythema and edema of the perianal region as a first sign (-Fig. 7).

Perianal contact dermatitis may progress to create multiple vesicles, oozing, and erosion surrounding cutis ani. Itching is the main symptom and leads to the itch-scratch-lichenification cycle of perianal skin. Fingers and nails should be examined. Eczema can be found spread by the itch-scratch phenomenon over body's intertriginous zones and joints. Frequently, different areas may be affected simultaneously or sequentially as shown in - Fig. 8. Lichenification is present in chronic eczema.

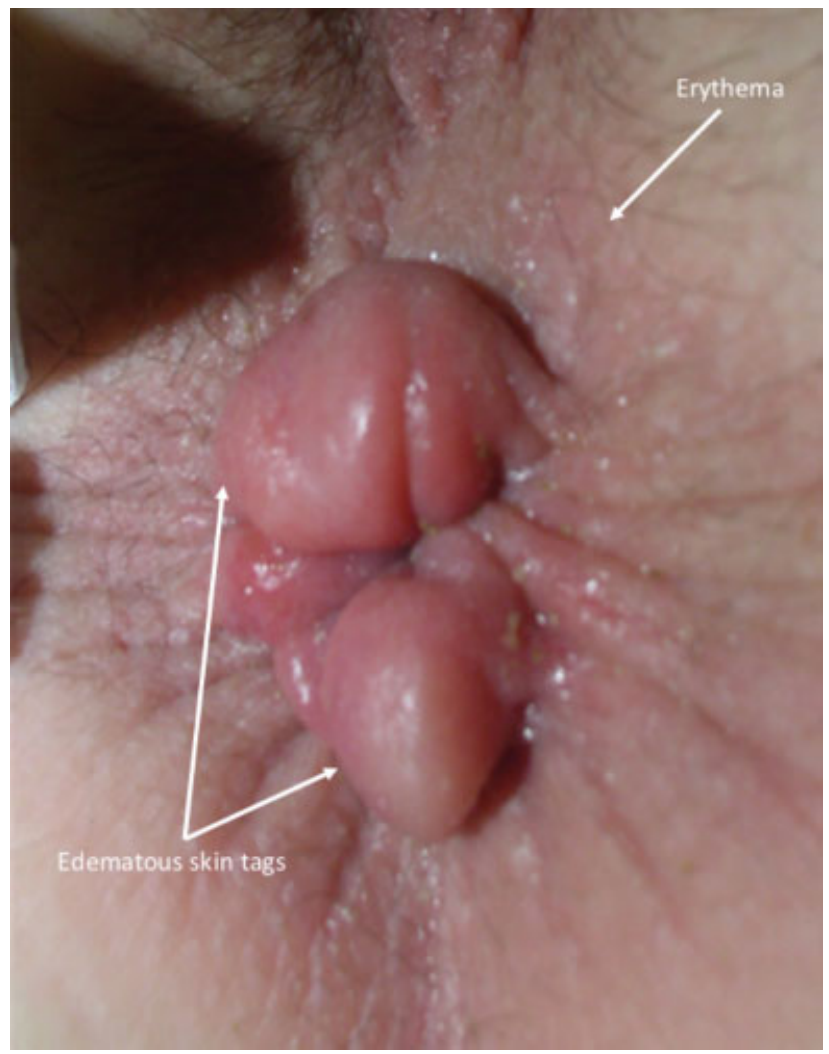

Fig. 7 Erythema and reactive edema in the perianal eczema (This image is provided courtesy of X. Delgadillo.)
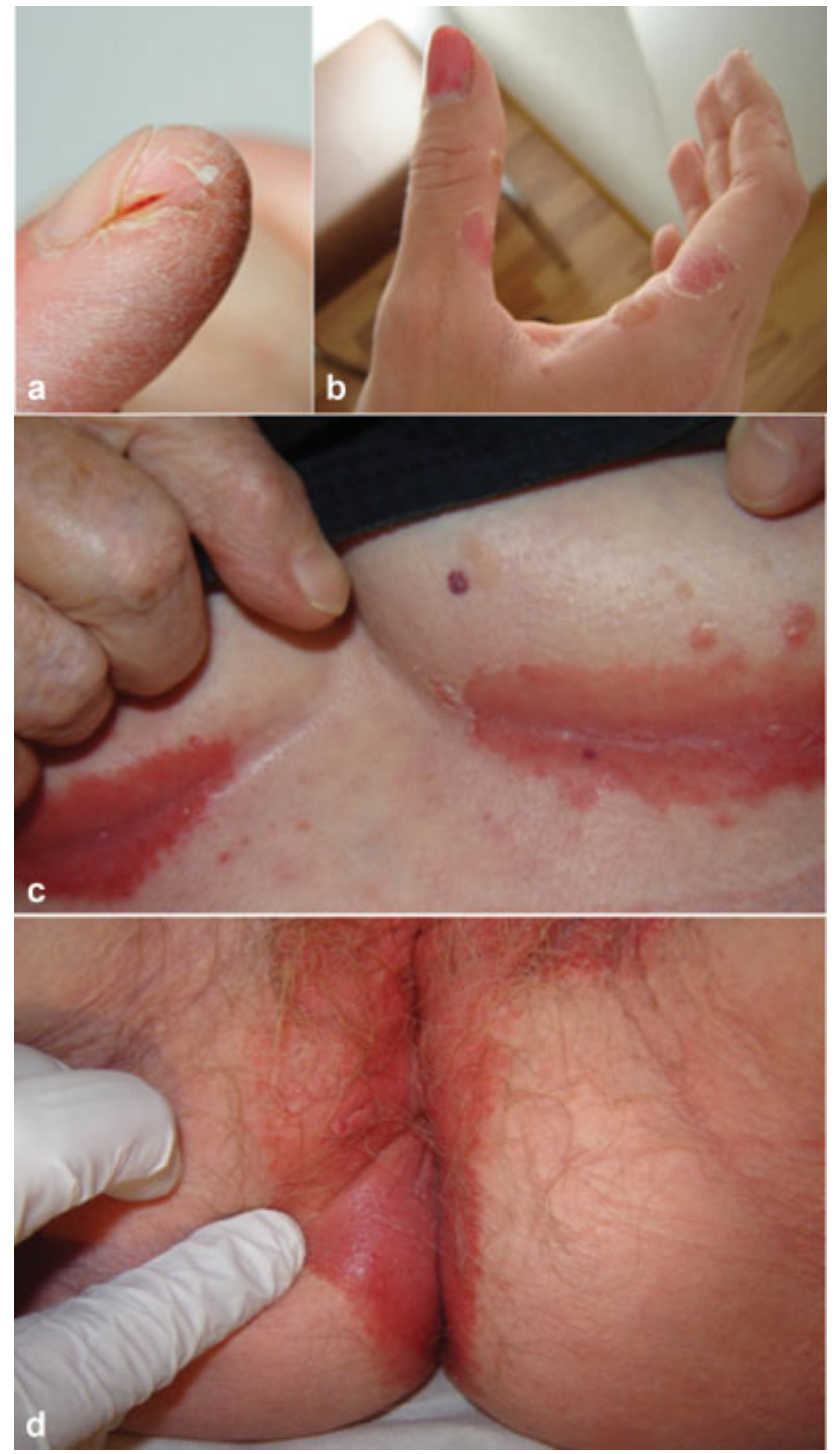

Fig. 8 Synchronous body itch-scratch-lichenification cycle in case of chronic dermatitis involving (a) nails, (b) fingers, (c) inframammary folds, and (d) perianal region. (These images are provided courtesy of X. Delgadillo.)

\section{A Practical Clinical Approach to Perianal Dermatitis}

Evaluation follows the same principles described for idiopathic pruritus ani. Interrogatories regarding specific irritants and other potentially contributing behaviors are indicated. Examination from head to toe is important. When pustules are present, their content should be examined by Gram's strain. Cultures for Candida should be performed on fungal media. Bacterial cultures are generally not helpful and unnecessary. Viral examination should be done particularly on the presence of visible erosions. In cases of well-delimited plaques, biopsy is necessary to exclude dermatosis.

The fundamental premise in treatment is the exclusion of the inciting agent. ${ }^{8}$ Failure to accomplish this goal may result nonresolution, recurrence, and ultimately in chronic dermatitis. The best local therapy in case of acute contact dermatitis is to clean the perianal region with tepid water. Hydrotherapy to perianal region can be accomplished with sitz baths, 
bidet, or showers particularly with a portable shower head. This practical common remedy is supported by the observation on Greek infants who do not get diaper dermatitis because these infants are cleaned under running water. ${ }^{7-9}$ Patients are encouraged to employ some form of hydrotherapy following bowel movements. Nonporous clothing and garments that keep the buttocks held tightly in apposition are discouraged. Instead of rubbing with toilet paper, drying can be done with a hair dryer. Lotions are preferred over ointments. Every attack of pruritus should be "treated" by a cool sitz bath. Topical steroids are widely used in dermatitis in other regions. Their use in the anal region is generally not indicted except in cases of acute allergic contact dermatitis.

Long-term topical application of fluorinated steroids may induce side effects. Hydrophobic barrier creams are also helpful for long-term prevention of recurrences. Antihistamines can be helpful in controlling pruritus ani. ${ }^{9}$ Fluorine steroids should be avoided. If they are absolutely necessary, their use should be limited to a strict minimum, and the rules of treatment with topical steroids must be strictly observed. Local steroid therapy should be applied once a day because of the phenomenon of tachyphylaxis of the skin. Abrupt discontinuation of topical steroids will be followed by a flare-up. Candida albicans infection frequently occurs after prolonged application of topical corticoids. It is treated with topical imidazole.

Chronic contact dermatitis can develop when the removal of the offending agent no longer provides expected relief. The skin will be lichenified (thickened) leading to excoriations with or without either hyper- or hypopigmented lesions surrounding the affected region.
Conflict of Interest

None.

\section{References}

1 Mallon E. Dermatosis of perineal and perianal skin. In: Griffiths C, Barker J, Bleiker T, Chalmers R, Creamer D eds. Rook's Textbook of Dermatology, 9th ed. Vol. 3. West Sussex, UK: Wiley Blackwell; 2010:113.1-113.33

2 Farouk R, Duthie GS, Pryde A, Bartolo DC. Abnormal transient internal sphincter relaxation in idiopathic pruritus ani: physiological evidence from ambulatory monitoring. Br J Surg 1994;81 (04):603-606

3 Allan A, Ambrose NS, Silverman S, Keighley MR. Physiological study of pruritus ani. Br J Surg 1987;74(07):576-579

4 Lysy J, Sistiery-Ittah M, Israelit Y, et al. Topical capsaicin-a novel and effective treatment for idiopathic intractable pruritus ani: a randomised, placebo controlled, crossover study. Gut 2003;52 (09):1323-1326

5 Suys E. Randomized study of topical tacrolimus ointment as possible treatment for resistant idiopathic pruritus ani. J Am Acad Dermatol 2012;66(02):327-328

6 Samalavicius NE, Poskus T, Gupta RK, Lunevicius R. Long-term results of single intradermal $1 \%$ methylene blue injection for intractable idiopathic pruritus ani: a prospective study. Tech Coloproctol 2012;16(04):295-299

7 Morris-Jones R, Robertson SJ, Ross JS, White IR, McFadden JP, Rycroft RJ. Dermatitis caused by physical irritants. $\mathrm{Br}$ J Dermatol 2002;147(02):270-275

8 Bourke J, Coulson I, English J; British Association of Dermatologists Therapy Guidelines and Audit Subcommittee. Guidelines for the management of contact dermatitis: an update. $\mathrm{Br} J$ Dermatol 2009;160(05):946-954

9 McGirt LY, Martins CR. Dermatologic diagnoses in the perianal area. Clin Colon Rectal Surg 2004;17(04):241-245 